\title{
Transfert de marqueur entre particules alimentaires au cours du transit digestif chez le lapin. Méthode d'estimation basée sur la granulométrie fécale
}

\author{
T. Gidenne avec la collaboration technique de B. Loupiac et \\ I. Chavichvili
}

INRA, Laboratoire de recherches sur l'élevage du lapin, BP 27, 31326 Castanet-Tolosan Cedex, France

(reçu le 9-2-1988, accepté le 16-9-1988)

Résumé - Cinq lapines adultes (néo-zélandaise blanche $\mathrm{x}$ californienne) ont reçu ad libitum pendant 10 jours un aliment complet granulé contenant de la luzerne déshydratée dont les particules de taille comprise entre 0,05 et $0,5 \mathrm{~mm}$ sont marquées à l'aide d'une terre rare : l'ytterbium (Yb). Pour estimer la proportion de marqueur susceptible de quitter son site initial de fixation, nous avons mesuré la quantité d'ytterbium présente dans les fèces sur les particules de taille supérieure à 0,5 $\mathrm{mm}$. Les fèces ont été collectées en totalité durant les 3 jours suivants.

Pour cette étude, la technique de tamisage des fèces a été adaptée de celle mise au point pour l'aliment. Elle présente toutefois une faible répétabilité dans le cas des grosses particules $(T>1 \mathrm{~mm}$ ) quantitativement peu représentées. La moitié des particules alimentaires $(52 \%)$ ont une taille inférieure à $0,05 \mathrm{~mm}$. Dans les fèces, seules les grosses particules $(T>1 \mathrm{~mm})$ sont peu représentées $(1,5 \%)$, tandis que les proportions des autres classes de particules varient entre 20 et $33,5 \%$.

Après la granulation, l'Yb initialement fixé sur des particules de luzerne, de tailles comprises entre 0,5 et $0,05 \mathrm{~mm}$, est en fait présent pour plus de $40 \%$ sur de fines particules $(T<0,05 \mathrm{~mm}$ ). De ce fait, on ne peut mesurer que les quantités minima et maxima d'Yb ayant migré sur des grosses particules ( $T>0,5 \mathrm{~mm}$ ), soit respectivement : 2,5 et $8,9 \%$ de l'Yb ingéré. En tenant également compte d'une migration vers de petites particules, environ $10 \%$ de l'Yb serait susceptible de quitter son site initial de fixation au cours de son transit dans le tube digestif du lapin.

terres rares - marqueur — granulométrie fécale — lapin

Summary - Marker transfer between feed particles during their passage through the digestive tract of rabbits. Methods of estimation based on faecal particle size. Five adult rabbits (New Zealand White $x$ Californian) were fed ad libitum for 10 days a pelleted feed compound containing dehydrated lucerne whose particles $(0.05-0.5 \mathrm{~mm}$ in size) were marked with a rare earth, i.e. ytterbium (Yb). In order to estimate the proportion of marker liable to leave its initial site of binding, we measured the amount of ytterbium present on faeces particles larger than $0.5 \mathrm{~mm}$. The total amount of faeces was collected during the following 3 days.

The faeces screening technique used in this study was adapted from that applied to feed. However, the repeatability was low for the large particles (>1 mm), whose number was small. Half 
of the digesta particles (52\%) exhibited a smaller size than $0.05 \mathrm{~mm}$. The proportion of large particles $(>1 \mathrm{~mm})$ in the faeces was low $(1.5 \%)$ while that of the other particle classes ranged from 20 $33.5 \%$.

After pelleting, more than $40 \%$ Yb initially bound to luzerne particles measuring 0.5 to $0.05 \mathrm{~mm}$, was present on fine particles $(<0.05 \mathrm{~mm}$ ). Accordingly, only minima and maxima amounts of $\mathrm{Yb}$ having migrated on large particles (>0.5 mm) could be measured, i.e. 2.5 and $8.9 \%$, respectively of the ingested $\mathrm{Yb}$. Taking also into account a migration towards small particles, about $10 \% \mathrm{Yb}$ was liable to leave its initial site during its passage through the digestive tract of the rabbit.

rare earths - marker - faecal particle size - rabbit

\section{Introduction}

Les études de transit et de flux digestif nécessitent l'emploi de marqueurs fiables. Parmi les nombreuses qualités demandées à tout marqueur particulaire (Kotb et Luckey, 1972) une des plus importantes est la résistance de la liaison marqueursupport alimentaire envers les agents digestifs. Les terres rares sont fréquemment utilisées en raison de leurs propriétés d'adsorption sur la fraction particulaire de l'aliment, que ce soit pour des études réalisées chez le ruminant (Ellis et Huston, 1968; Poncet et al., 1986) ou chez le lapin (Laplace et al., 1974). Mais certaines terres rares (cérium, samarium) peuvent quitter leur site initial de fixation durant leur séjour dans le rumen (Hartnell et Satter, 1979). Ce phénomène paraît accentué pour des milieux digestifs acides (Allen et Van Soest, 1982; Crooker et al., 1982). Or, chez le lapin, le pH stomacal varie de 1,5 à 2,5 (Gidenne et Lebas, 1984); une migration de marqueurs tels que l'ytterbium est envisageable, malgré les précautions prises lors de leur fixation sur l'aliment. II est donc important d'estimer ce phénomène, en particulier pour étudier simultanément le transit de plusieurs matières premières d'un même aliment, à l'aide de plusieurs marqueurs tels que ytterbium et cérium (Gidenne et al., 1987).
Notre travail vise à estimer la quantité d'ytterbium susceptible de se détacher de son support initial au cours du transit digestif du lapin. Nous avons fixé de l'ytterbium sur des particules de foin dont la taille est comprise entre 0,5 et $0,05 \mathrm{~mm}$. Puis nous avons dosé la quantité d'Yb présente dans les fèces sur des tailles de particules supérieures à $0,5 \mathrm{~mm}$. En effet, dans ce cas, l'Yb ne peut provenir que d'un transfert, et non d'une dégradation des particules par les phénomènes digestifs. Cela nécessite une détermination de la granulométrie de l'aliment et des fèces. Dans le cas de l'aliment, ce type de technique a déjà été mise au point (Lebas et Loupiac, non publié), mais elle n'est pas applicable dans le cas des fèces. La première partie de notre travail a donc été consacrée à la mise au point d'une technique de tamisage applicable aux fèces dures du lapin.

\section{Matériel et Méthodes}

\section{Animaux et alimentation}

Cinq lapines adultes, de race néo-zélandaise blanche $\times$ californienne, ont reçu à volonté, durant 10 jours, un aliment complet granulé à base de luzerne déshydratée $(89,9 \%$ de $M O$, $17,5 \%$ de MAT, $10,6 \mathrm{MJ}$ d'ED/kg MS, $37,2 \%$ de NDF, $23 \%$ de ADF). Pendant les 3 derniers jours, une récolte totale des fèces a été réalisée. Les fèces sont pesées fraîches puis stockées à $-18^{\circ} \mathrm{C}$. 


\section{Marquage de l'aliment}

L'aliment contient un marqueur de transit, spécifique de la fraction particulaire : l'ytterbium (Yb). Cette terre rare est fixée sur de la luzerne déshydratée, préalablement débarrassée de ses constituants cellulaires solubles à l'aide d'un détergent neutre (Uden et al., 1980). Le marquage est réalisé selon le principe de Ellis et Beever (1985) : trempage du foin dans une solution de $\mathrm{Yb}$ durant $24 \mathrm{~h}$, suivi d'un rinçage soigneux sous l'eau du robinet, puis d'un trempage dans une solution chélatrice (acide citrique $0,7 \mathrm{M} /$ moles d'Yb fixé) en vue de solubiliser les molécules d'Yb faiblement liées aux particules. Environ $20 \mathrm{~g}$ de foin ainsi préparé $(8,6 \mathrm{mg} \mathrm{Yb/g} \mathrm{MS)}$ ont été broyés (broyeur centrifuge Retsch) à la grille de $0,5 \mathrm{~mm}$, puis tamisés à sec (tamiseuse Retsch 3D). Seules les particules de foin $(25 \mathrm{~g})$ dont la taille est comprise entre 0,5 et $0,05 \mathrm{~mm}$ sont retenues, puis incorporées après un prémélange aux matières premières broyées $(12 \mathrm{~kg}$ ) constituant l'aliment. L'ensemble est ensuite aggloméré sous forme de granulé de $3,5 \mathrm{~mm}$ de diamètre.

\section{Tamisage de l'aliment et des fèces}

L'aliment est tamisé par voie humide selon la technique de Lebas et Lamboley (1989, sous presse). Les tamis suivants sont utilisés pour l'aliment et les fèces : $1,0,5,0,315$ et $0,05 \mathrm{~mm}$. Pour simplifier le langage, nous emploierons le terme de taille de particules "T $T$ " pour exprimer une classe de particules retenues sur un tamis de maille donnée et non retenues par un tamis aux mailles plus grandes.

Tamisage des fèces en vue de la détermination de leur teneur en $\mathrm{Yb}$.

Dans le cas des fèces de lapin, aucune technique de tamisage n'a jusqu'alors été proposée. Aussi, dans le but d'effectuer un dosage d'Yb sur les différentes classes de particules, nous avons appliqué une technique visant à ne pas modifier les concentrations en $\mathrm{Yb}$ sur chaque taille de particules. Le principe est voisin de celui appliqué aux aliments :

- trempage des fèces fraîches $(30 \mathrm{~g})$ dans $600 \mathrm{ml}$ d'eau à température ambiante durant $12 \mathrm{~h}$;

- désagrégation manuelle des fèces;

- passage des fèces dans une cuve à ultrasons (fréquence $40 \mathrm{kHz}$, puissance $200 \mathrm{~W}$ ) pendant 2 périodes de $10 \mathrm{~min}$. Les ultrasons entrainent la formation de microbulles. Leur implosion à la surface des crottes permet de détacher les particules périphériques sans modifier leur structure;

- tamisage par voie humide durant $10 \mathrm{~min}$.

Une granulométrie a été réalisée sur un échantillon de fèces provenant de chaque animal. Après examen visuel, il s'avère que quelques agglomérats de particules subsistent sur les tamis de $1 \mathrm{~mm}$. Ces agglomérats proviennent de fines particules non désagrégées à la suite du prétraitement ou incluses dans des poils. En vue de doser l'ytterbium, nous avons donc prélevé un échantillon de chaque classe de particules en prenant garde de ne pas retenir ces agglomérats.

\section{Tamisage des fèces pour quantification du nombre de particules.}

Dans le but de mesurer plus précisément la quantité de particules isolées dans chaque tamis, nous avons appliqué une autre technique de prétraitement de l'échantillon évitant la formation d'agglomérats :

- $30 \mathrm{~g}$ de fèces fraîches sont trempées durant $1 \mathrm{~h}$ dans l'eau à $50^{\circ} \mathrm{C}$;

- trempage durant $1 \mathrm{~h}$ dans l'eau à ébullition. Les crottes sont alors désagrégées en quasitotalité;

- l'échantillon est ensuite soumis à un bain d'ultrasons, durant $10 \mathrm{~min}$.

Nous avons également modifié légèrement la technique de tamisage. Un tamis à maille de $2 \mathrm{~mm}$ est ajouté, dans le but de retenir les poils inclus dans les crottes dures. Le tamisage débute sous aspersion d'eau manuelle, afin de vérifier que seuls les poils sont retenus sur le tamis de $2 \mathrm{~mm}$. L'échantillon est ensuite tamisé durant $10 \mathrm{~min}$ avec variations de la fréquence d'agitation de la tamiseuse. Cette mesure est répétée 4 fois pour chaque échantillon individuel de fèces.

Comme dans le cas de l'aliment, la quantité de particules fécales inférieures à $0,05 \mathrm{~mm}$ est estimée par différence entre le poids initial de l'échantillon et le cumul des poids de chaque classe de particules supérieures à $0,05 \mathrm{~mm}$.

\section{Dosage de l'ytterbium}

Un gramme de fèces ou d'aliment sec est calciné durant $5 \mathrm{~h}$ à $550^{\circ} \mathrm{C}$. L'ytterbium est extrait à chaud des cendres par l'acide nitrique $\left(\mathrm{HNO}_{3}\right.$ $1,5 \mathrm{~N}+\mathrm{KCl} 0,2 \%$; puis il est dosé par spectrophotométrie d'absorption atomique (PerkinElmer 2380). Ces dosages ont concerné un échantillon de fèces par animal et un échan- 
tillon d'aliment. lls ont été réalisés sur l'échantillon avant son tamisage puis sur chaque classe de particules.

L'ytterbium a été dosé également dans l'eau du bain d'ultrasons pour 3 échantillons de luzerne marquée afin de vérifier l'innocuité des ultrasons sur le détachement d'Yb de son support. L'échantillon est alors atomisé dans un micro-four (et non par une flamme) afin d'augmenter la sensibilité de l'analyse (1 ppb).

\section{Analyse statistique}

L'analyse statistique des données issues du tamisage est effectuée par analyse de variance à 2 facteurs de classification : effet de l'individu et effet de la répétition de la mesure (programme Anvarm et Calea de la programmathèque Amance 81; Bachacou et al., 1981).

\section{Résultats}

\section{Granulométrie de l'aliment et des fèces}

L'aliment contient beaucoup de particules très fines $(52 \%)$ non retenues sur le tamis
$0,05 \mathrm{~mm}$; les proportions de particules retenues sur les autres tamis sont comprises entre 8 et $17 \%$ (Fig. 1). Les fèces ne présentent pas cette disproportion entre classes de particules : seules les particules dont $\mathrm{T}$ est supérieure à $1 \mathrm{~mm}$ sont très peu représentées $(1,5 \%$ en moyenne), les proportions des autres classes variant de 20 à $33,5 \%$.

Les proportions moyennes de particules fécales de taille inférieure à $0,05 \mathrm{~mm}$ et supérieure à $0,5 \mathrm{~mm}$ ont été très variables entre animaux (CV : 15 à $30 \%$ ). Nous n'avons pas observé d'effet "répétition" significatif sur la mesure des tailles de particules. Mais la répétabilité de la mesure est faible pour les classes extrêmes $(55 \%$ pour $T>1 \mathrm{~mm}$ en moyenne sur les 5 individus), elle est de $7,4 \%$ en moyenne pour $0,05<\mathrm{T}<0,315 \mathrm{~mm}$.

La collecte totale des fèces a permis de préciser les quantités de matière sèche excrétées pour chaque taille de particules, et de comparer cette répartition

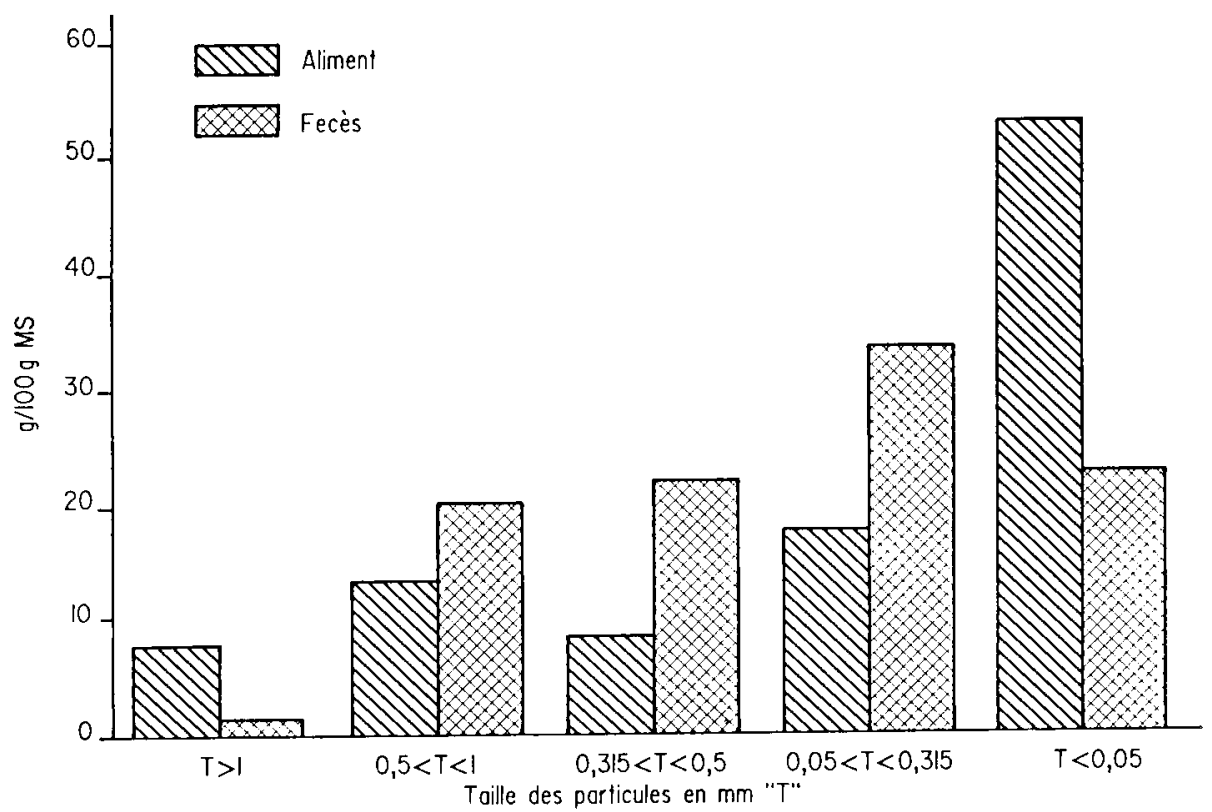

Fig. 1. Granulométrie de l'aliment et des fèces correspondantes chez le lapin. 


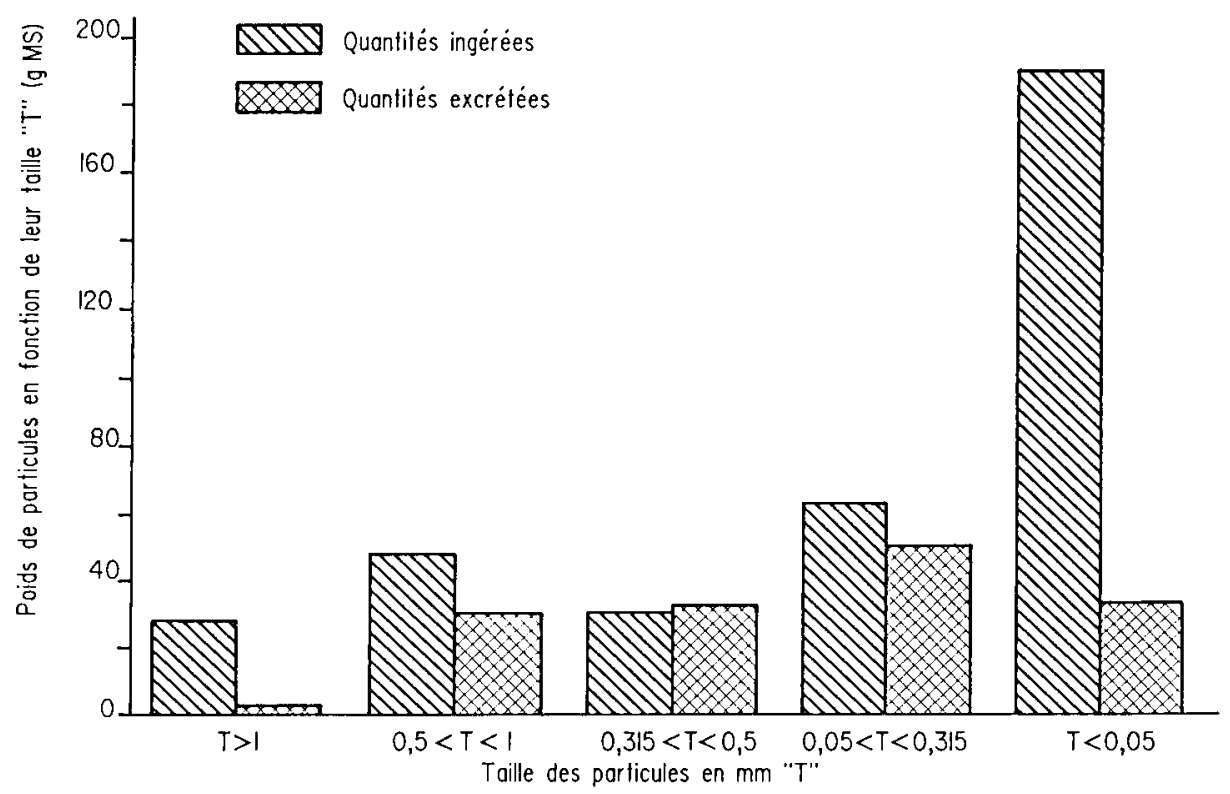

Fig. 2. Comparaison des quantités de particules ingérées et excrétées en fonction de leur taille T.

granulométrique à celle caractérisant la MS ingérée (Fig. 2). Moins de 10\% des particules alimentaires de taille supérieure à $1 \mathrm{~mm}$ sont encore présentes dans les fèces. Dans le cas des autres classes de particules, il est difficile d'évaluer l'impor-

Tableau I. Répartition de l'ytterbium dans l'aliment et les fèces selon les tailles de particules.

\begin{tabular}{|c|c|c|c|c|c|c|}
\hline & Total & $T>1 \mathrm{~mm}$ & $0,5<<1 \mathrm{~mm}$ & $0,315<<0,5$ & $0,05<<0,31$ & $<0,05$ \\
\hline \multicolumn{7}{|l|}{$\mathrm{Yb}(\mu \mathrm{g} / \mathrm{g}$ de $\mathrm{MS})$} \\
\hline Aliment $(n=3)$ & $\begin{array}{r}17,9 \\
\pm 1,2\end{array}$ & $\begin{array}{r}3,8 \\
\pm 0,2\end{array}$ & $\begin{array}{r}6,3 \\
\pm 0,9\end{array}$ & $\begin{array}{r}13,8 \\
\pm 0,1\end{array}$ & $\begin{array}{r}46,1 \\
\pm 0,7\end{array}$ & ND \\
\hline Fèces $(n=5)$ & $\begin{array}{r}43,4 \\
\pm 2,3\end{array}$ & $\begin{array}{c}31 \\
\pm 2,6\end{array}$ & $\begin{array}{r}19,5 \\
\pm 2,2\end{array}$ & $\begin{array}{r}23,2 \\
\pm 3,3\end{array}$ & $\begin{array}{r}39,5 \\
\pm 5,5\end{array}$ & ND \\
\hline \multicolumn{7}{|l|}{$q Y b(\%){ }^{1}$} \\
\hline Yb ingéré (\%) & 100 & 1,7 & 4,7 & 6,6 & 44,8 & $42,2^{2}$ \\
\hline $\begin{array}{l}\text { Yb excrété (\%) } \\
(x \pm \sigma) n=5\end{array}$ & 100 & $\begin{array}{r}0,83 \\
\pm 0,45\end{array}$ & $\begin{array}{r}8,1 \\
\pm 1,6\end{array}$ & $\begin{array}{c}11 \\
\pm 2,2\end{array}$ & $\begin{array}{r}31,6 \\
\pm 6,4\end{array}$ & $\begin{array}{l}48,4 \\
\pm 92\end{array}$ \\
\hline
\end{tabular}

$1: q \mathrm{Yb}$ : quantité d'Yb exprimée en \% du total excrété.

2 : ces valeurs sont obtenues par différence entre la quantité totale d'Yb dans l'échantillon et la somme des quantités d'Yb déterminées dans les différentes classes de particules $(>0,05 \mathrm{~mm})$.

ND : non déterminé. 
tance de la dégradation due au phénomène digestif, en raison de l'arrivée de particules de classes de taille supérieure. II faut toutefois remarquer l'écart très important entre la quantité de très fines particules $(T<0,05 \mathrm{~mm}$ ) dans l'aliment (190 g/MS) et celle retrouvée dans les fèces $(33,7 \mathrm{~g} / \mathrm{MS})$.

Effet des ultrasons sur le détachement de Yb de son support

A partir de 3 échantillons de luzerne (contenant en moyenne $1,1 \mathrm{mg}$ de $\mathrm{Yb}$ ), nous détectons dans l'eau du bain après 2 périodes de $10 \mathrm{~min}$ d'ultrasons entre 1,6 et $2,15 \mu \mathrm{g} \mathrm{d}^{\prime} \mathrm{Yb}$, soit en moyenne $0,16 \pm 0,04 \% d^{\prime} Y b$ fixé initialement sur l'échantillon de luzerne.

Répartition de l'ytterbium dans l'aliment et les fèces selon la taille des particules

Le taux de récupération de l'ytterbium, calculé par collecte totale des fèces pendant 3 jours consécutifs, a été en moyenne de $98,7 \pm 3,3 \%(n=5)$; ce marqueur est donc indigestible.

Le principe adopté était d'obtenir un marquage des particules de taille comprise entre 0,5 et 0,05 mm. Dans l'aliment, ces classes de particules ont présenté effectivement la concentration en $\mathrm{Yb}$ la plus élevée (Tableau I). Mais nous avons également trouvé des quantités d'Yb importantes dans les autres classes de particules. Ainsi, 51,4\% seulement de l'ytterbium contenu dans l'aliment sont réellement fixés sur des particules de 0,5 à $0,05 \mathrm{~mm}$; alors que $42,2 \%$ de ce marqueur sont fixés sur des particules très fines $(T<0,05 \mathrm{~mm}$ ) (Tableau $\mathrm{I}$ ).

La quantité maximum d'Yb transférée (Tf maxi) sur des particules de taille supérieure à celles marquées initialement est représentée par la quantité totale d'Yb trouvée dans les fèces sur des particules de taille supérieure à $0,5 \mathrm{~mm}$. Nous considérons alors comme nul l'apport d'Yb provenant des particules alimentaires de même taille $(T>0,5 \mathrm{~mm}$ ) non dégradées par les phénomènes digestifs. Dans notre expérience, Tf maxi équivaut à $8,9 \%$ de la totalité de Yb ingéré.

La quantité minimum d'Yb (Tf mini) transférée sur des particules de taille supérieure à $0,5 \mathrm{~mm}$ équivaut à la quantité mesurée dans les fèces sur ces particules, défalquée de la quantité mesurée dans l'aliment, sur les particules de même taille. Dans notre cas, Tf mini équivaut à $2,5 \%$ de l'Yb ingéré.

\section{Discussion et conclusion}

Tamisage des fèces : qualité de la méthode

Après examen visuel, il semble qu'il n'y ait aucune variation de la mesure due à la présence (en quantité variable) de particules plus fines que les mailles d'un tamis; en particulier, dans le cas de particules supérieures à $1 \mathrm{~mm}$, cette fraction contenait à l'issue des premiers essais de tamisage des agglomérats de fines particules ou des "réseaux" de poils emprisonnant des particules de toutes tailles. Notre méthode de tamisage de fèces est donc correcte au plan qualitatif. Mais les coefficients de variations (CV) intra-individuel calculés à partir de 4 mesures par individu sont très élevés dans le cas des classes de particules quantitativement peu représentées. Par exemple, le CV pour les particules $>1 \mathrm{~mm}$ est en moyenne de $55 \%$. Or, ces particules isolées à partir de $30 \mathrm{~g}$ de fèces fraîches représentent en fait moins de $1 \mathrm{~g}$ de matière sèche. Ces variations intra-individuelle semblent donc dues, au moins pour une part, à un problème de quantité 
d'échantillon. II serait souhaitable d'accroître la quantité de fèces initiale pour diminuer l'imprécision des mesures sur les faibles quantités.

Cette technique de tamisage des fèces permet donc une analyse quantitative de la distribution des tailles de particules (Fig. 2). Jusqu'alors, peu d'auteurs ont abordé ce problème chez le lapin. Uden et Van Soest (1982) indiquent une granulométrie similaire entre crottes dures et crottes molles, sans préciser la distribution des tailles de particules. Enfin, Bjornhag (1972) indique que 55\% des particules prélevées dans le côlon distal ont une taille inférieure à $0,315 \mathrm{~mm}$, en accord avec nos résultats.

\section{Répartition de l'ytterbium dans l'aliment et les fèces}

Dans le cas de l'aliment, l'ytterbium était en principe fixé, en totalité, sur des particules dont $T$ était compris entre 0,5 et $0,05 \mathrm{~mm}$. II s'avère en fait, qu'après granulation de l'aliment, plus de $40 \%$ de I'Yb sont présents sur des particules non retenues par le tamis de $0,05 \mathrm{~mm}$. Ceci n'est pas imputable à la technique de tamisage puisque après dispersion aux ultrasons, moins de $0,2 \%$ de l'Yb fixé est présent dans l'eau du bain.

La granulation réduirait donc notablement la taille des particules; de plus, elle provoquerait la réagglomération de particules, puisque $6,4 \%$ de l'Yb sont présents sur des particules de tailles supérieures à $0,5 \mathrm{~mm}$. Lebas (1986) constate aussi une modification du profil granulométrique d'un aliment, entre le stade de broyage et de granulation. La présence d'Yb en quantité importante sur les particules de moins de $0,05 \mathrm{~mm}$ peut également être expliquée par un tamisage imparfait du foin marqué. En effet, ce dernier a été réalisé à sec; par conséquent, il est possible qu'une quantité importante de très fines particules $(T<0,05 \mathrm{~mm})$ n'ait pas traversé le tamis de $0,5 \mathrm{~mm}$, par effet électrostatique entre particules. Un profil granulométrique de l'aliment broyé, avant et après agglomération, serait nécessaire pour juger précisément cet effet sur la réduction de taille des particules.

Nous ne pouvons pas estimer le transfert de marqueur vers les fines particules; par contre, notre méthode permet de mesurer le transfert minimum et maximum d'Yb vers des particules de tailles supérieures : respectivement 2,5 et $8,9 \%$ de la quantité totale d'Yb. En effet, les particules alimentaires contenant de l'Yb peuvent être dégradées au cours du transit digestif et être présentes sur des particules fécales de tailles inférieures à $0,05 \mathrm{~mm}$. De plus, la présence d'Yb déprime la digestion de la matière sèche (Teeter et al., 1984). Mais nous ne connaissons pas l'importance de ces phénomènes.

II apparaît donc vraisemblable que le transfert de marqueur sur des particules plus grosses que celles initialement marquées soit de l'ordre de $5 \%$ de l'Yb total ingéré. Dans l'hypothèse où les phénomènes de transfert vers de fines particules atteignent les mêmes proportions, au total environ $10 \%$ de l'Yb serait susceptible de migrer sur des particules autres que celles sur lesquelles il était préalablement fixé. Teeter et al. (1984) estiment in vitro que la migration $d^{\prime} \mathrm{Yb}$ au niveau du rumen concernerait 0,1 à $0,6 \%$ de l'Yb fixé initialement par heure d'incubation. Hartnell et Satter (1979) estiment qu'après $24 \mathrm{~h}$ d'incubation, moins de $1 \%$ du marqueur (cérium, samarium) est susceptible de se détacher d'un foin. Mais ces auteurs montrent aussi que le phénomène de migration est plus important lorsque la matière première contenant le marqueur est moins fibreuse (céréales, pulpes, etc.). 
En conclusion, la technique de fixation de l'Yb sur des fourrages, utilisée chez le ruminant, paraît relativement bien adaptée au lapin. Toutefois, une quantité non négligeable $(\approx 10 \%)$ de l'Yb serait susceptible de quitter son site initial de fixation durant son séjour dans le tube digestif. Le marqueur déplacé de son site est alors susceptible de s'adsorber à nouveau sur la même matière première et sur d'autres composants de la ration. Ainsi, lorsque l'on emploie plusieurs marqueurs pour mesurer le temps de séjour de plusieurs composants d'un même aliment (Poncet et al., 1986), les phénomènes de migration de marqueurs peuvent abaisser les écarts mesurables entre matières. Cela est particulièrement important pour des matières au transit différent (fourrages et produits plus "concentrés" tels que céréales, tourteaux) pour lesquelles les capacités d'adsorption des terres rares sont différentes. II conviendrait donc d'appliquer une méthode de fixation des marqueurs en fonction de la matière considérée, pour obtenir une liaison marqueur-support stable vis-à-vis des différents agents digestifs : acidité des contenus, dégradation enzymatique, flore microbienne, etc.

\section{Références}

Allen M.S. \& Van Soest P.J. (1982) Comparative affinities of rare earth elements for plant cell-walls. J. Anim. Sci. 55 (Suppl. 1), 403

Bachacou M.T., Masson J.P. \& Millier C. (1981) Manuel de la Programmathèque Statistique Amance 81. INRA Publication

Bjornhag G. (1972) Separation and delay of contents in the rabbit colon. Swed. J. Agric. Res. 2, 125-136

Crooker B.A., Clark J.H. \& Shanks D.R. (1982) Rare earth elements as markers for rate of passage measurements of individual feedstuffs through the digestive tract of ruminants. J. Nutr. 112, 1353-1366
Ellis W.C. \& Huston J.E. (1968) $144 \mathrm{Ce}-144 \mathrm{Pr}$ as a particulate digesta flow marker in ruminants. J. Nutr. 95, 67-78

Ellis W.C. \& Beever D.E. (1985) Methods for binding rare earths to specific feed particles. In : Techniques in Particle Size Analysis of Feed and Digestion in Ruminants (P.M. Kennedy, Ed.), Can. Soc. Anim. Sci., Edmonton, occ. publ. $n^{\circ} 1,154-165$

Gidenne T. \& Lebas F. (1984) Evolution circadienne du contenu digestif chez le lapin en croissance. Relation avec la cæcotrophie. 3e Congr. Mon. Cuniculture, Rome, Vol. 2, 494501

Gidenne T., Poncet C. \& Gomez L. (1987) Effet de l'addition d'un concentré riche en fibres dans une ration à base de foin, distribuée à deux niveaux alimentaires chez la lapine adulte. 1. Temps de séjour des aliments. Repr. Nutr. Develop. 27, 733-743

Hartnell G.F. \& Satter L.D. (1979) Extent of particulate marker (samarium, lanthanum and cerium) movement from one digesta particle to another. J. Anim. Sci. 48, 375

Kotb R.A. \& Luckey T.D. (1972) Markers in nutrition. Nutr. Abstr. Rev. 42, 813-845

Laplace J.P., Lebas F. \& Rioperez J. (1974) Le transit digestif chez le lapin. I. Utilisation du cérium 141 : étude méthodologique et descriptive. Ann. Zootech. 23, 555-576

Lebas F. (1986) Effet du broyage des matières premières avant agglomération de deux aliments à taux variable de constituants membranaires : digestibilité et performances zootechniques. $2^{\mathrm{es}}$ Journ. Rech. Cunic. France, 10-11 déc., Paris, communic. $n^{\circ} 9$

Lebas F. \& Lamboley B. (1989) Ann. Zootech. (sous presse)

Poncet C., Gomez L. \& Michalet-Doreau B. (1987) Temps de séjour comparé du foin, du son de blé et de la pulpe de betterave, d'une ration mixte distribuée à deux niveaux alimentaires à des moutons et des taurillons. Reprod. Nutr. Dévelop. 27, 219-220

Teeter R.G., Owens F.N. \& Mader T.L. (1984) Ytterbium chloride as a marker in the rumen. J. Anim. Sci. 58, 465-473

Uden P., Colucci E.P. \& Van Soest P.J. (1980) Investigation of chromium, cerium and cobalt as markers in digesta. Rate of passage studies. J. Sci. Food Agric. 31, 625-632

Uden P. \& Van Soest P.J. (1982) The determination of digesta particle size in some herbivores. Anim. Feed. Sci. Tech. 7, 35-44 